\title{
Radial velocities, metallicities, and distances of Cepheids in M31 and M33
}

\author{
Amy D. Forestell, Thomas G. Barnes III, Christopher Sneden \\ The University of Texas at Austin, Department of Astronomy, \\ 1 University Station, C1400, Austin, TX 78712-0259, USA \\ Thomas J. Moffett \\ Purdue University, Physics Department, 1396 Physics Bldg., West \\ Lafayette, IN 47907-1396, USA
}

\begin{abstract}
Cepheid variable stars are fundamental to the calibration of the extragalactic distance scale. We present radial velocities, preliminary metallicities, and preliminary surface brightness distances of Cepheids in Local Group galaxies M31 and M33 using data obtained with the High Resolution Spectrograph on the Hobby-Eberly Telescope at McDonald Observatory. These data are the first step toward surface brightness distance measurements and metallicity calibrations of these extragalactic Cepheids.
\end{abstract}

\section{Introduction}

Cepheid variable stars are widely used as distance calibrators because they are luminous, easy to discover through their large amplitude and characteristic light curves, and obey a Period-Luminosity (P-L) relationship. This P-L relation is calibrated through four techniques of distance measurement: statistical parallax, cluster zero age main sequence fitting, trigonometric parallax, and the surface brightness technique. The method used in this study is the surface brightness (Baade-Wesselink) technique as developed by Barnes et al. (1977), which compares the varying angular diameter, measured from color (surface brightness) and apparent magnitude, with the variation of physical radius, measured from integration of the radial velocity curve, to obtain a quasi-geometric distance. Nordgren et al. (2002) have found interferometric angular diameters of nearby Cepheids and have thus geometrically calibrated the surface brightness, color relationship. Reddening has almost no effect on the distance and the metallicity of each Cepheid can be measured to test possible dependencies. Therefore this independent method is useful in checking systematic errors of other methods.

Stellar evolutionary models (Bono et al. 2000; Alibert et al. 1999) and stellar pulsation theory (Fiorentino 2002) predict a dependence of the P-L relation on metallicity. We aim to constrain this possible metallicity issue. 

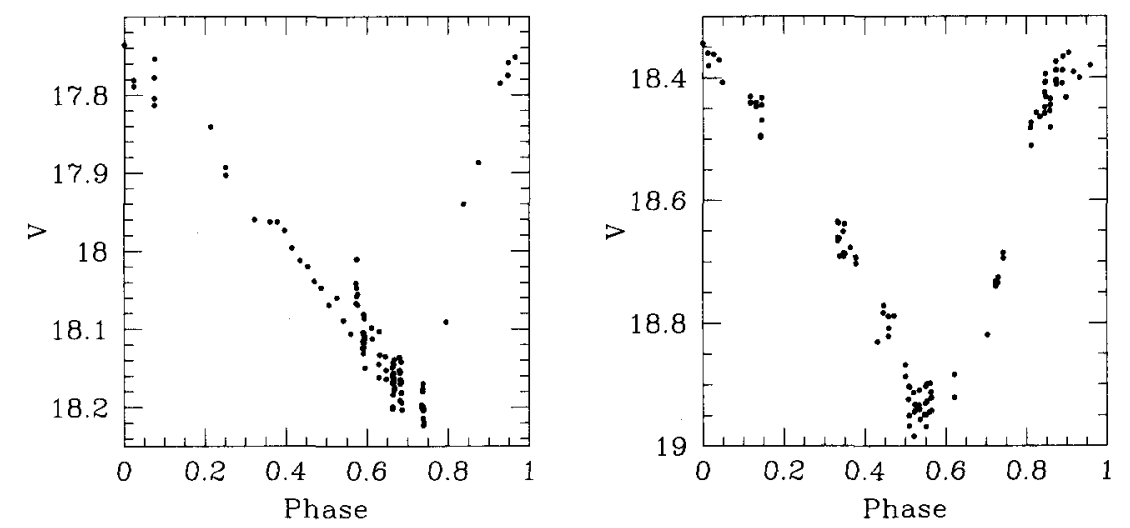

Figure 1. $\quad V$ Light curve for V2203_D31F and V22665_D33B from DIRECT data.

\section{Observations and data reduction}

We use echelle spectra of one Cepheid in M31 and one Cepheid in M33 obtained with the fiber-fed High Resolution Spectrograph on the 9.2-m Hobby-Eberly Telescope at McDonald Observatory. These Cepheids have magnitudes in the range $V=17.8-19.0$ and our $R=15000$ spectra have peak $\mathrm{S} / \mathrm{N}$ of order $5-10$ in 1200-3000 s integrations. The echelle spectra have 37 orders over two chips covering the wavelength range $4800-6800 \AA$. The spectra were reduced using standard IRAF routines.

Photometric light curves in $V$ and $I$ were obtained from the DIRECT database (Fig. 1). Kaluzny et al. (1998) give an explanation of their photometry, which is available for download at http://cfa-www.harvard.edu/ "kstanek/DIRECT/. $(V-I)_{C}$ color is transformed to $(V-R)_{J}$ using the precepts of Cousins (1981).

\section{Radial velocities}

Using the Tonry \& Davis (1979) cross-correlation method as utilized in the IRAF task 'fxcor' we have measured the radial velocities of Cepheids V2203_D31F and V22665_D33B with respect to Galactic standard stars of known velocity. We use Chauvenet's criterion, an iterative sigma clipping algorithm, to select the useful orders and achieve an internal precision of $\pm 1.4 \mathrm{kms}^{-1}$ over a maximum of 22 orders. The reliability of our technique was tested and confirmed using two radial velocity standard stars observed on the same night. The measured radial velocity curves (Fig. 2) agree with expectations from the light variation. 

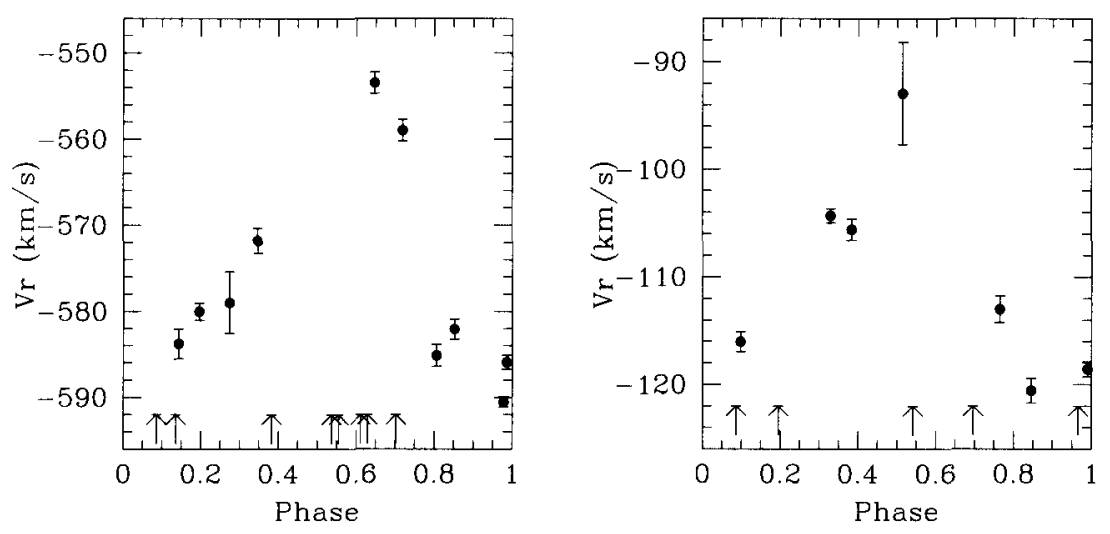

Figure 2. V2203_D31F and V22665_D33B radial velocity curves. The unreduced observations are indicated by arrows to illustrate the full range of our observations.

\section{Metallicities}

We measured metallicities of our Cepheids by matching synthetic spectra to our observed spectra. We produced synthetic spectra with the current version of the LTE line analysis code, MOOG, of Sneden (1973), using ATLAS9 stellar atmosphere models and the Kurucz line list. A $\chi^{2}$ calculation was used to select the best-fitting metallicity model.

The technique was tested on a sky spectrum using a Holweger-Muller solar atmosphere. We measure $[\mathrm{Fe} / \mathrm{H}]$ of -0.08 dex with a standard error of the mean across 22 orders of 0.026 dex. The line list and atmosphere models were not optimized for the sun, so we are encouraged by these results. In addition our technique was tested on CTIO 4-m data of Cepheid T Mon obtained by Luck et al. (1998) and is consistent with their metallicity results.

Preliminary $\chi^{2}$ matches on our Cepheids give an $[\mathrm{Fe} / \mathrm{H}]$ with an internal precision of approximately $\pm 0.1 \mathrm{dex}$. At this time imprecision in the sky subtraction is preventing an accurate metallicity determination. We are exploring several options to improve the sky subtraction.

\section{Distances}

The distances of our Cepheid variable stars are measured with a Bayesian statistical analysis of the surface brightness (Baade-Wesselink) method as devised by Barnes et al. (2003) (see also Barnes et al., these proceedings). With our partial radial velocity curves we measure the following preliminary distance moduli. For V22665_D33B using $A_{V}=0.794$ and $E(B-V)=0.230$ we measure ( $V$ $\left.M_{V}\right)=23.84(+0.32-0.37)$. Using $A_{V}=0.397$ and $E(B-V)=0.115$ we measure $\left(V-M_{V}\right)=23.87(+0.39-0.48)$, demonstrating independence from reddening. The Hubble Key Project (Freedman et al. 2001) finds $\left(V-M_{V}\right)=24.62 \pm 0.15$, which includes a metallicity correction. For V2203_D31F using $A_{V}=0.754$ 
and $E(B-V)=0.219$ we find $\left(V-M_{V}\right)=25.26(+0.46-0.58)$. The Hubble Key Project finds $\left(V-M_{V}\right)=24.48 \pm 0.12$. These are first order results and will become more precise with improved measures of photometry, colors, velocities, and metallicity corrections.

\section{Conclusions}

We have measured the radial velocities and preliminary distances and metallicities of one Cepheid in M31 and one Cepheid in M33. The techniques that we have employed for extracting this information from faint sources are successful, and future work will improve their precision. With our results for these and future Cepheids we will obtain a check on the Cepheid distance scale and its possible dependence on metallicity.

Acknowledgments. We thank the DIRECT researchers for their assistance in target selection. This work is supported through NSF grants AST9986817 (TGB) and AST-9987347. (TJM).

\section{References}

Alibert, Y., Baraffe, I., Hauschildt, P., Allard, F. 1999, A\&A, 344, 551

Barnes, T.G., Dominy, J.F., Evans, D.S., Kelton, P.W., Parsons, S.B., Stover, R.J. 1977, MNRAS, 178, 661

Barnes, T.G., Jefferys, W.H., Berger, J.O., Mueller, P.J., Orr, K., Rodriguez, R. 2003, ApJ, 592, 539

Bono, G., Caputo, F., Cassisi, S., Marconi, M., Piersanti, L., Tornambè, A. 2000, ApJ, 543, 955

Cousins, A.W.J. 1981, Mon. Not. Astr. Soc. South Africa, 40, 37

Fiorentino, G., Caputo, F., Marconi, M., Musella, I. 2002, ApJ, 576, 402

Freedman, W.L., et al. 2001, ApJ, 553, 47

Kaluzny, J., Stanek, K.Z., Krockenberger, M., Sasselov, D.D., Tonry, J.L., Mateo, M. 1998, AJ, 115, 1016

Luck, R.E., Moffett, T.J., Barnes, T.G., Gieren, W.P. 1998, AJ, 115, 605

Nordgren, T.E., Lane, B.F., Hindsley, R.B., Kervella, P. 2002, AJ, 123, 3380

Sneden, C. 1973, ApJ, 184, 839

Tonry, J., Davis, M. 1979, AJ, 84, 1511 